\title{
ON A NONLINEAR STOCHASTIC INTEGRAL EQUATION OF THE HAMMERSTEIN TYPE
}

\author{
W. J. PADGETT
}

Abstract. A nonlinear stochastic integral equation of the Hammerstein type in the form

$$
x(t ; \omega)=h(t ; \omega)+\int_{s} k(t, s ; \omega) f(s, x(s ; \omega)) d \mu(s)
$$

is studied where $t \in S, a \sigma$-finite measure space with certain properties, $\omega \in \Omega$, the supporting set of a probability measure space $(\Omega, A, P)$, and the integral is a Bochner integral. A random solution of the equation is defined to be a second order vector-valued stochastic process $x(t ; \omega)$ on $S$ which satisfies the equation almost certainly. Using certain spaces of functions, which are spaces of second order vector-valued stochastic processes on $S$, and fixed point theory, several theorems are proved which give conditions such that a unique random solution exists.

1. Introduction. The purpose of this note is to study the existence and uniqueness of a random solution of a nonlinear stochastic integral equation of the Hammerstein type of the form

$$
x(t ; \omega)=h(t ; \omega)+\int_{S} k(t, s ; \omega) f(s, x(s ; \omega)) d \mu(s),
$$

where

(i) $S$ is a locally compact metric space with metric $d$ defined on $S \times S$ and $\mu$ is a complete $\sigma$-finite measure defined on the collection of Borel subsets of $S$;

(ii) $\omega \in \Omega$, where $\Omega$ is the supporting set of the probability measure space $(\Omega, A, P)$;

(iii) $x(t ; \omega)$ is the unknown vector-valued random variable for each $t \in S$;

(iv) $h(t ; \omega)$ is the stochastic free term defined for $t \in S$;

(v) $k(t, s ; \omega)$ is the stochastic kernel defined for $t$ and $s$ in $S$; and

(vi) $f(t, x)$ is a vector-valued function of $t \in S$ and $x$.

The integral in equation (1.1) is interpreted as a Bochner integral [12].

Received by the editors July 3, 1972 and, in revised form, August 31, 1972.

AMS (MOS) subject classifications (1970). Primary 60H20, 45G99; Secondary 60G99.

Key words and phrases. Stochastic Hammerstein integral equation, nonlinear stochastic integral equation, random solutions, second order processes.

(c) American Mathematical Society 1973 
Further assumptions concerning the functions in (1.1) will be stated in $\S 2$.

The equation (1.1) is a generalization of stochastic integral equations studied by Padgett and Tsokos [9], Tsokos [11], and Anderson [1]. Also, equation (1.1) is a stochastic version of the deterministic integral equations which were investigated by Petryshyn and Fitzpatrick [10], Browder and Gupta [5], Browder, de Figueiredo, and Gupta [6], among others.

In order to investigate the stochastic integral equation (1.1), we will define several spaces of functions which are spaces of second order vector-valued stochastic processes on $S$ and will use certain aspects of the "theory of admissibility" of Banach spaces as introduced into the study of integral equations by Corduneanu [7] and the methods of "probabilistic functional analysis" [3].

2. Preliminaries. We will further assume that $S$ is the union of a countable family of compact subsets $\left\{C_{n}\right\}$ having the properties that $C_{1} \subset C_{2} \subset C_{3} \subset \cdots$ and that for any other compact set in $S$ there is a $C_{i}$ which contains it [2].

We define $C=C\left(S, L_{2}(\Omega, A, P)\right)$ to be the space of all continuous functions from $S$ into the space $L_{2}(\Omega, A, P)$ with the topology of uniform convergence on compacta. That is, for each fixed $t \in S, x(t ; \omega)$ is a vectorvalued random variable such that

$$
\|x(t ; \omega)\|_{L_{2}(\Omega, A, P)}^{2}=\int_{\Omega}|x(t ; \omega)|^{2} d P(\omega)<\infty .
$$

It may be noted that $C\left(S, L_{2}(\Omega, A, P)\right)$ is a locally convex space [12, pp. 24-26] whose topology is defined by the countable family of seminorms given by

$$
\|x(t ; \omega)\|_{n}=\sup _{t \in C_{n}}\|x(t ; \omega)\|_{L_{2}(\Omega, A, P)}, \quad n=1,2, \cdots .
$$

Moreover, $C\left(S, L_{2}(\Omega, A, P)\right)$ is complete relative to this topology since $L_{2}(\Omega, A, P)$ is complete.

We further define $B C=B C\left(S, L_{2}(\Omega, A, P)\right)$ to be the Banach space of all bounded continuous functions from $S$ into $L_{2}(\Omega, A, P)$ with norm

$$
\|x(t ; \omega)\|_{B C}=\sup _{t \in S}\|x(t ; \omega)\|_{L_{2}(\Omega, A, P)} .
$$

The space $B C \subset C$ is the space of all second order vector-valued stochastic processes defined on $S$ which are bounded and continuous in mean-square.

We will consider the functions $h(t ; \omega)$ and $f(t, x(t ; \omega))$ to be in the space $C\left(S, L_{2}(\Omega, A, P)\right)$. With respect to the stochastic kernel we assume that for each pair $(t, s), k(t, s ; \omega) \in L_{\infty}(\Omega, A, P)$ and denote the norm by

$$
\|k(t, s ; \omega)\|\|=\| k(t, s ; \omega) \|_{L_{\infty}(\Omega, A, P)}=\underset{\omega \in \Omega}{\operatorname{ess} \sup }|k(t, s ; \omega)| .
$$


Also, we will suppose that $k(t, s ; \omega)$ is such that

$$
\|k(t, s ; \omega)\|\|\cdot\| x(s ; \omega) \|_{L_{2}(\Omega, A . P)}
$$

is $\mu$-integrable with respect to $s$ for each $t \in S$ and $x(s ; \omega)$ in $C\left(S, L_{2}(\Omega, A, P)\right)$, and that there exists a real-valued function $G$ defined $\mu$-a.e. on $S$ so that $G(s)\|x(s ; \omega)\|_{L_{2}(\Omega . A, P)}$ is $\mu$-integrable and, for each pair $(t, s) \in S \times S$,

$$
\|k(t, u ; \omega)-k(s, u ; \omega)\| \cdot\|x(u ; \omega)\|_{L_{2}(\Omega, A, P)} \leqq G(u)\|x(u ; \omega)\|_{L_{2}(\Omega, A, P)}
$$

$\mu$-a.e. Further, for almost all $s \in S, k(t, s ; \omega)$ will be continuous in $t$ from $S$ into $L_{\infty}(\Omega, A, P)$.

We now define the integral operator $T$ on $C\left(S, L_{2}(\Omega, A, P)\right)$ by

$$
(T x)(t ; \omega)=\int_{S} k(t, s ; \omega) x(s ; \omega) d \mu(s),
$$

where the integral is a Bochner integral. From the conditions on $k(t, s ; \omega)$, we have that for each $t \in S,(T x)(t ; \omega) \in L_{2}(\Omega, A, P)$ and that $(T x)(t ; \omega)$ is continuous in mean square by Lebesgue's dominated convergence theorem. That is, $(T x)(t ; \omega) \in C\left(S, L_{2}(\Omega, A, P)\right)$.

LEMMA 2.1. The linear operator $T$ defined by equation (2.1) is continuous from $C\left(S, L_{2}(\Omega, A, P)\right)$ into itself.

Proof. Note that $C\left(S, L_{2}(\Omega, A, P)\right)$ is a Fréchet space with metric $d^{*}$ defined by the Fréchet combination of the sequence of seminorms $\|\cdot\|_{n}$, $n=1,2, \cdots$.

Define the sequence of linear operators $\left\{T_{M}\right\}, M=1,2, \cdots$, by

$$
\left(T_{M} x\right)(t ; \omega)=\int_{C_{M}} k(t, s ; \omega) x(s ; \omega) d \mu(s) .
$$

Hence, as $M \rightarrow \infty$ we have $\left(T_{M} x\right)(t ; \omega) \rightarrow(T x)(t ; \omega)$.

Let $\left\{x_{j}(t ; \omega)\right\}$ be a sequence of functions converging to $x(t ; \omega)$ in $C\left(S, L_{2}(\Omega, A, P)\right)$. Then by definition of the seminorms, for each $M$

$$
\begin{aligned}
\|\left(T_{M} x\right)(t ; \omega) & -\left(T_{M} x_{j}\right)(t ; \omega) \|_{n} \\
& \leqq \sup _{t \in C_{n}} \int_{C_{M}}\|k(t, s ; \omega)\| \cdot\left\|x(s ; \omega)-x_{j}(s ; \omega)\right\|_{L_{2}(\Omega, A, P)} d \mu(s) .
\end{aligned}
$$

Since $\left\|x(s ; \omega)-x_{j}(s ; \omega)\right\|_{L_{2}(\Omega, A, P)} \rightarrow 0$ uniformly on the compact set $C_{M}$, for $\varepsilon>0$ there exists a positive integer $N_{M}$ such that $j \geqq N_{M}$ implies

$$
\left\|\left(T_{M} x\right)(t ; \omega)-\left(T_{M} x_{j}\right)(t ; \omega)\right\|_{n}<\varepsilon \sup _{t \in C_{n}} \int_{C_{M}}\|k(t, s ; \omega)\| d \mu(s) .
$$


Now, by the conditions on $k(t, s ; \omega)$, there exists a constant $K_{n}$ such that $\| k\left(t, s ; \omega\|\| K_{n}\right.$ for all $t \in C_{n}$ and almost all $s$. Hence, for $j \geqq N_{M}$

$$
\left\|\left(T_{M} x\right)(t ; \omega)-\left(T_{M} x_{j}\right)(t ; \omega)\right\|_{n}<\varepsilon K_{n} \mu\left(C_{M}\right) .
$$

Since convergence in every seminorm is equivalent to convergence in the metric $d^{*},\left(T_{M} x_{j}\right)(t ; \omega)$ converges to $\left(T_{M} x\right)(t ; \omega)$ in $C\left(S, L_{2}(\Omega, A, P)\right)$ for each $M$. Therefore, by [8, p. 54], $T$ is continuous from $C\left(S, L_{2}(\Omega, A, P)\right)$ into itself.

Let $B$ and $D$ be Banach spaces. The pair $(B, D)$ is said to be admissible with respect to a linear operator $T$ if $T(B) \subset D$.

LEMMA 2.2. If $T$ is a continuous linear operator from $C\left(S, L_{2}(\Omega, A, P)\right)$ into itself and $B, D \subset C\left(S, L_{2}(\Omega, A, P)\right)$ are Banach spaces stronger than $C\left(S, L_{2}(\Omega, A, P)\right)$ such that $(B, D)$ is admissible with respect to $T$, then $T$ is continuous from $B$ into $D$.

The lemma follows from the closed-graph theorem.

From Lemmas 2.1 and 2.2 it follows that $T$ defined by equation (2.1). is a bounded linear operator from $B$ into $D$.

By a random solution of the equation (1.1) we will mean a function $x(t ; \omega)$ in $C\left(S, L_{2}(\Omega, A, P)\right)$ which satisfies the equation $P$-a.e.

3. Existence of a random solution. We now present theorems concerning the existence and uniqueness of a random solution of the equation (1.1).

THEOREM 3.1. We consider the stochastic integral equation (1.1) subject to the following conditions:

(i) $B$ and $D$ are Banach spaces stronger than $C\left(S, L_{2}(\Omega, A, P)\right)$ such that $(B, D)$ is admissible with respect to the integral operator defined by equation (2.1);

(ii) $x(t ; \omega) \rightarrow f(t, x(t ; \omega))$ is an operator from the set

$$
Q(\rho)=\left\{x(t ; \omega): x(t ; \omega) \in D,\|x(t ; \omega)\|_{D} \leqq \rho\right\}
$$

into the space $B$ satisfying the Lipschitz condition

$$
\|f(t, x(t ; \omega))-f(t, y(t ; \omega))\|_{B} \leqq \lambda\|x(t ; \omega)-y(t ; \omega)\|_{D}
$$

for $x(t ; \omega), y(t ; \omega) \in Q(\rho)$, where $\rho$ and $\lambda$ are constants;

(iii) $h(t ; \omega) \in D$.

Then there exists a unique random solution of (1.1) in $Q(\rho)$, provided $\lambda K<1$ and

$$
\|h(t ; \omega)\|_{D}+K\|f(t, 0)\|_{B} \leqq \rho(1-\lambda K),
$$

where $K$ is the norm of $T$. 
Proof. Define the operator $U$ from $Q(\rho)$ into $D$ by

$$
(U x)(t ; \omega)=h(t ; \omega)+\int_{S} k(t, s ; \omega) f(s, x(s ; \omega)) d \mu(s) .
$$

Then from the conditions of the theorem

$$
\begin{aligned}
\|(U x)(t ; \omega)\|_{D} & \leqq\|h(t ; \omega)\|_{D}+K\|f(t, x(t ; \omega))\|_{B} \\
& \leqq\|h(t ; \omega)\|_{D}+K\|f(t, 0)\|_{B}+K \lambda\|x(t ; \omega)\|_{D} \leqq \rho .
\end{aligned}
$$

Hence, $(U x)(t ; \omega) \in Q(\rho)$.

Now, for $x(t ; \omega), y(t ; \omega) \in Q(\rho)$ we have by condition (ii) that

$$
\begin{aligned}
\|(U x)(t ; \omega)- & (U y)(t ; \omega) \|_{D} \\
& =\left\|\int_{S} k(t, s ; \omega)[f(s, x(s ; \omega))-f(s, y(s ; \omega))] d \mu(s)\right\|_{D} \\
& \leqq K\|f(t, x(t ; \omega))-f(t, y(t ; \omega))\|_{B} \\
& \leqq \lambda K\|x(t ; \omega)-y(t ; \omega)\|_{D} .
\end{aligned}
$$

Since $\lambda K<1, U$ is a contraction on $Q(\rho)$.

Therefore, by Banach's fixed point theorem there exists a unique $x^{*}(t ; \omega) \in Q(\rho)$ which is a fixed point of $U$, that is, $x^{*}(t ; \omega)$ is the unique random solution of equation (1.1).

A similar theorem may be obtained when $f$ is a nonlinear contraction on $Q(\rho)[4]$.

THEOREM 3.2. Assume that the stochastic integral equation (1.1) satisfies the following conditions:

(i) same as Theorem 3.1(i);

(ii) $x(t ; \omega) \rightarrow f(t, x(t ; \omega))$ is an operator from the set $Q(\rho)$ into the space $B$ satisfying

$$
\|f(t, x(t ; \omega))-f(t, y(t ; \omega))\|_{B} \leqq \phi\left(\|x(t ; \omega)-y(t ; \omega)\|_{D}\right)
$$

for $x(t ; \omega), y(t ; \omega) \in Q(\rho)$, where $\phi$ is a real-valued continuous function such that $\phi(s)<s$ for $s>0$;

(iii) $h(t ; \omega) \in D$.

Then there exists a unique random solution of (1.1) in $Q(\rho)$, provided $K \leqq 1$ and $\|h(t ; \omega)\|_{D}+K\|f(t, 0)\|_{B} \leqq \rho(1-K)$, where $K$ is the norm of $T$.

The proof of Theorem 3.2 is similar to that of Theorem 3.1 except that the fixed point theorem of Boyd and Wong [4] is used.

The following is a useful application of Theorem 3.1. 
COROLlaRY 3.1. Suppose the stochastic integral equation (1.1) satisfies the following conditions:

(i) $\sup _{t \in S} \int_{S}\|k(t, s ; \omega)\| d \mu(s)<\infty$;

(ii) $f(t, x)$ is a continuous function of $t \in S$ uniformly in $x$ such that for $\|x(t ; \omega)\|_{B C},\|y(t ; \omega)\|_{B C} \leqq \rho$

$$
\|f(t, x(t ; \omega))-f(t, y(t ; \omega))\|_{L_{2}(\Omega, A, P)} \leqq \lambda\|x(t ; \omega)-y(t ; \omega)\|_{L_{2}(\Omega, A, P)}
$$

for each $t \in S$, where $\lambda$ and $\rho$ are constants;

(iii) $h(t ; \omega)$ is a bounded continuous function from $S$ into $L_{2}(\Omega, A, P)$.

Then there exists a unique random solution of equation (1.1), provided $\sup _{t \in S} \int_{S}\|k(t, s ; \omega)\| d \mu(s), \lambda$, and $\|f(t, 0)\|_{B C}$ are sufficiently small.

Proof. We must show that condition (i) implies that the pair $(B C, B C)$ is admissible with respect to the integral operator $T$ defined by equation (2.1). Let $x(t ; \omega) \in B C\left(S, L_{2}(\Omega, A, P)\right)$. Then by the properties of the Bochner integral

$$
\begin{aligned}
\|(T x)(t ; \omega)\|_{L_{2}(\Omega, A, P)} & \leqq \int_{S}\|k(t, s ; \omega) x(s ; \omega)\|_{L_{2}(\Omega, A, P)} d \mu(s) \\
& \leqq \sup _{t \in S}\|x(t ; \omega)\|_{L_{2}(\Omega, A, P)} \int_{S}\|k(t, s ; \omega)\| d \mu(s) \\
& \leqq\|x(t ; \omega)\|_{B C} \sup _{t \in S} \int_{S}\|k(t, s ; \omega)\| d \mu(s) .
\end{aligned}
$$

Hence, $(T x)(t ; \omega) \in B C\left(S, L_{2}(\Omega, A, P)\right)$, that is, $(B C, B C)$ is admissible with respect to $T$.

Conditions (ii)-(iii) clearly imply that conditions (ii)-(iii) of Theorem 3.1 hold. Thus, by Theorem 3.1, there exists a unique random solution of equation (1.1).

Other corollaries of Theorems 3.1 and 3.2 may be obtained by choosing different Banach spaces contained in the space $C\left(S, L_{2}(\Omega, A, P)\right)$ and different conditions on $f$ and $k$.

\section{REFERENCES}

1. M. W. Anderson, Stochastic integral equations, Ph.D. Dissertation, University of Tennessee, Nashville, Tenn., 1966.

2. R. F. Arens, A topology for spaces of transformations, Ann. of Math. (2) 47 (1946), 480-495. MR 8, 165.

3. A. T. Bharucha-Reid, On the theory of random equations, Proc. Sympos. Appl. Math., vol. 16, Amer. Math. Soc., Providence, R.I., 1964, pp. 40-69. MR 32 \#6498.

4. D. W. Boyd and J. S. W. Wong, On nonlinear contractions, Proc. Amer. Math. Soc. 20 (1969), 458-464. MR 39 \#916.

5. F. E. Browder and C. P. Gupta, Monotone operators and nonlinear integral equations of Hammerstein type, Bull. Amer. Math. Soc. 75 (1969), 1347-1353. MR 40 \#3381. 
6. F. E. Browder, D. G. de Figueiredo and C. P. Gupta, Maximal monotone operators and nonlinear integral equations of Hammerstein type, Bull. Amer. Math. Soc. 76 (1970), 700-705. MR 42 \#8347.

7. C. Corduneanu, Problèmes globaux dans la théorie des équations intégrales de Volterra, Ann. Mat. Pura Appl. (4) 67 (1965), 349-363. MR 32 \#331.

8. N. Dunford and J. T. Schwartz, Linear operators. I: General theory, Pure and Appl. Math., vol. 7, Interscience, New York, 1958. MR 22 \#8302.

9. W. J. Padgett and C. P. Tsokos, A random Fredholm integral equation, Proc. Amer. Math. Soc. 33 (1972), 534-542.

10. W. V. Petryshyn and P. M. Fitzpatrick, New existence theorems for nonlinear equations of Hammerstein type, Trans. Amer. Math. Soc. 160 (1971), 39-63. MR 43 \#6784.

11. C. P. Tsokos, On a stochastic integral equation of the Volterra type, Math. Systems Theory 3 (1969), 222-231. MR 40 \#949.

12. K. Yosida, Functional analysis, Die Grundlehren der math. Wissenschaften, Band 123, Academic Press, New York; Springer-Verlag, Berlin, 1965. MR 31 \#5054.

Department of Mathematics, University of South Carolina, Columbia, South Carolina 29208 